\title{
3 Research Square \\ Efficacy and safety of simple exercise interventions for men with osteoporosis - a prospective randomized controlled trial
}

Franca Maria Natalie Genest

Ludwig-Maximilians-Universitat Munchen Medizinische Fakultat https://orcid.org/0000-0001-62334805

\section{Sarah Lindström}

Julius-Maximilians-Universitat Wurzburg Medizinische Fakultat

\section{Sophia Scherer}

Julius-Maximilians-Universitat Wurzburg Medizinische Fakultat

\section{Michael Schneider}

Julius-Maximilians-Universitat Wurzburg Medizinische Fakultat

Lothar Seefried ( $\sim$ l-seefried.klh@uni-wuerzburg.de)

University of Wuerzburg https://orcid.org/0000-0003-1154-3388

\section{Research article}

Keywords: Osteoporosis, Sarcopenia, Resistance Training, Whole Body Vibration, Spinal Orthosis, Qi Gong

Posted Date: April 30th, 2020

DOl: https://doi.org/10.21203/rs.3.rs-24837/v1

License: (c) (1) This work is licensed under a Creative Commons Attribution 4.0 International License. Read Full License 


\section{Abstract}

Background: Aging is associated with progressive loss of musculoskeletal performance. Exercise interventions can improve physical function in the elderly but there is a paucity of comparative assessments in order to understand what specific goals can be achieved particularly with less demanding exercise interventions readily accessible for untrained men.

Methods: Prospective randomized, controlled, single center trial to compare efficacy and feasibility of four distinct exercise interventions for 6 months in men at risk for osteoporosis aged 65-90 years. Primary endpoint was change in isometric one repetition maximum force trunk strength for extension (TSE) and flexion (TSF) compared to baseline, secondary endpoints covered key parameters of geriatric functional assessment, including Handgrip Strength (HS), Chair-Rise-Test (CRT) Usual Gait Speed (UGS) and TimedUp-and-Go (TUG).

Results: Altogether 47 men (mean age $77+/-6.1$ years) were randomized to Resistance Training (RT, $n=11)$, Whole Body Vibration Exercise (WBV, $n=13)$, Qi Gong ( $Q G, n=10)$ and wearing a Spinal Orthosis $(S O, n=13)$. RT lead to significant improvements for TSE $(p=0.009)$ and TSF $(p=0.013)$. Vibration exercise caused sign. Progress in TSE $(p=0.014)$ and CRT $(p=0.005)$, the Spinal Orthosis improved CRT $(p=0.003)$ and Gait Speed ( $p=0.027)$, while the QG Group did not attain any sig. developments.

Subgroup analyses revealed most pronounced musculoskeletal progress in vulnerable patients (age $\geq 80$ years, pre-sarcopenia, multimorbidity $\geq 3$ chronic diseases). Irrespective of the type of exercise, participants $\geq 80$ years experienced significant gain in TSE $(p=0.029)$ and CRT $(p=0.017)$. Presarcopenic subjects (Skeletal muscle Index (SMI) $\left.\leq 10.75 \mathrm{~kg} / \mathrm{m}^{2}\right)$ improved in TSE $(p=0.003), C R T(p=0.001)$ and UGS $(p=0.016)$. Multimorbid participants achieved sig. gains in TSE $(p<0.001)$, TSF $(p=0.002)$, UGS $(p=0.036)$ and $H S(p=0.046)$.

Conclusions: Simple exercise interventions are feasible in elderly men with specific efficacy, i.e. improvements are attained in those tasks addressed with the training. Consequently, exercise strategies in elderly men should comprise multiple modalities to comprehensively cover various deficits. For vulnerable patients, any simple training appears beneficial.

\section{Background}

Osteoporosis and sarcopenia both are critical determinants for impaired health outcomes and functional decline in aging (1-6). Specifically, reduced bone quality and quantity along with decreased muscle mass and function elevate the risk for falls and fractures, entailing restrictions in mobility and subsequent morbidity and mortality (7). In addition to osteoporosis, sarcopenia is a critical factor of progressive immobility and functional decline with aging $(5,8)$. Sarcopenia is commonly defined by reduced muscle mass combined with reduced muscle strength and/or impaired physical performance $(9,10)$. Growing evidence supports the perception of sarcopenia and functional decline being critical factors of elevated fracture risk, commonly reflected in approaches to define a condition of osteosarcopenia $(11,12)$. Current 
literature confirms that individuals with both conditions are at higher risk for decreased mobility, general health issues and increased mortality $(8,13,14)$. In this context, functional decline and impaired mobility or dysmobility appear to be of particular clinical significance in aging men $(15,16)$.

Concurrently, growing scientific data propose exercise interventions as effective measures to mitigate or even partially reverse this age associated decay and prevent adverse outcomes (17-23). Importantly, different exercise modalities in that regard exhibit a very distinct profile regarding both feasibility in the elderly as well as efficacy in terms of achieving meaningful training results and overall improvements. However, a critical appraisal of available evidence also reveals that despite the high number of studies in the field, there is a paucity of comparative analyses of distinct intervention modalities in randomized controlled studies with prespecified outcome parameters $(24,25)$. Currently, machine-supported or augmented, professionally supervised resistance training is a well-established, reliable and effective exercise intervention strategy to regain muscle mass and strength in the elderly $(22,26-28)$ and can be considered gold standard. However, real world feasibility and sustainability of such an intervention is limited by costs, accessibility, adherence, and the motivation of untrained seniors to familiarize with this type of exercise modality. Hence, easy-accessible, technically simple training concepts requiring less personal engagement were attractive alternatives, particularly for previously non-athletic, mobilityrestricted individuals. There is data suggesting that low impact training modalities like e.g. group-based Qi Gong may be safe, widely accepted and still effective (26). Growing evidence also supports Whole Body Vibration as being both an effective as well as efficient and safe exercise modality in vulnerable patients at risk for muscular deficits (29-31). Furthermore, there is also literature reporting effectiveness of a specific trunk supporting, flexible back brace in terms of increasing muscular performance by regular use without the need for a specific workout routine. This Spinal orthosis is a back brace approved for supportive treatment of osteoporosis with kyphosis and/or back pain (long-term care). Considering wellknown difficulties with encouraging (not only) older people to attend regular workout sessions, this appears a low-threshold alternative, specifically for individuals severely limited with regards to performing conventional physical training (32-35).

So far there are no comparative data to advise which of these alternative exercise modalities is preferable regarding both efficacy in terms of different functional outcome parameters on the one hand as well as safety and feasibility including acceptance on the other hand. In addition, there is a need to understand to what extend these alternative exercise interventions can keep up with the gold standard of a professionally supervised conventional resistance exercise intervention program regarding functional outcome.

\section{Methods}

Study design: This is a prospective randomized controlled single center trial to compare efficacy, feasibility and safety of four different exercise modalities over 6 months in community-dwelling elderly men at risk for osteoporosis and sarcopenia. 
Study population: Main inclusion criteria included male subjects $\geq 65$ years with one of the following indicators of relevant osteoporosis: Preexisting DXA-Scan with T-Score $\leq-2.5$ at the hip or spine, previously diagnosed osteoporosis or ongoing osteoporosis treatment or 10-year fracture risk probability $\geq 20 \%$ according to applicable national guidelines. Exclusion criteria mirrored contraindications to Whole Body Vibration according to the manufacturer's specifications.

Participants were identified from a previous study evaluating coincidence and potential interference of osteoporosis and sarcopenia in a large, community-derived cohort of elderly men. Out of 507 participants evaluated in that former study, 268 had risk factors warranting further assessment for osteoporosis according to applicable national guidelines and were therefore invited for a comprehensive musculoskeletal exam, including assessment of clinical risk factors for osteoporosis, DXA measurement and laboratory workup $(36,37)$. Out of 113 who accepted that offer, 57 were willing to participate in this six months exercise intervention study with randomized treatment group allocation. Ten men canceled participation before starting the intervention. A total of 47 participants completed the study per protocol (see Fig. 1 for details).

Figure 1: Study population flow

\section{Exercise intervention}

Exercise interventions were supervised Resistance Training (RT) as a reference, Whole Body Vibration Exercise (WBV) as externally stimulated muscle activation, Qi Gong (QG) classes as a low impact training modality and wearing a Spinal orthosis (SO) as an effortless, almost passive modality.

Resistance training: Professionally supervised, individually adjusted conventional resistance training with special focus on trunk strength comprising 8 different exercises was conducted for 30min twice weekly in a high-level gym facility for professional athletes (Predia, Wuerzburg, Germany).

Qi Gong training: Instructed group sessions $2 \times 45 \mathrm{~min}$ per week comprising various exercises of coordinated body-posture, low-impact movements, breathing and meditation rather than muscle power and strength training.

Whole Body Vibration training: Supervised WBV training sessions on a side alternating vibration platform (Galileo ${ }^{\circledR}$, Novotec Medical GmbH, Pforzheim, Germany) with vibration frequencies up to $25.5 \mathrm{~Hz}$. Exercises focusing on trunk strength and lower extremities were performed for $2 x 20 \mathrm{~min}$ per week.

Spinal orthosis: Participants were provided with an off-the-shelf orthosis (Spinomed active, Medi GmbH, Bayreuth, Germany) customized at the beginning of the study. Subjects were requested to wear the back brace for at least 3h per every day throughout the study, preferentially while being active. Wearing time of the orthosis and tolerability had to be documented on a daily basis.

\section{Assessments and Endpoints}


Baseline assessment of physical performance was scheduled before starting exercise intervention, an interim evaluation was performed at 3 months and final assessment was scheduled after 6 months of training.

Primary endpoint was one repetition maximum force isometric measurement of trunk strength for extension (TSE) and flexion (TSF) on a stationary machine (Typus FPZ Systems developed by Schnell Ltd. Peutenhausen, Germany). Secondary endpoints comprised key diagnostic parameters of geriatric functional assessment. Handgrip Strength (HS) was measured with a handheld dynamometer (DynEx1, Akern srl, Florence, Italy) in a seated position, the elbow flexed at 90 degrees. Best performance value out of three per side was used for further analyses. The three components of the Short Physical Performance Battery (SPPB) including Chair-Rise-Test (CRT), Usual Gait Speed (UGS) and static Balance (SB) were assessed as described previously in literature $(38,39)$. For distinct analysis of individual test SB results were calculated summing up total standing time, i.e. max $10 \mathrm{sec}$ side-by-side, $10 \mathrm{sec}$ semi-tandem and $10 \mathrm{sec}$ tandem stance, yielding $30 \mathrm{sec}$ as max result for best performance. In addition, the Timed-Up and Go (TUG) (40), the 6 minute walk test (6MWT) and the Skeletal Muscle Index (SMI, skeletal muscle mass by height squared, $\mathrm{kg} / \mathrm{m}^{2}$ ) derived from Bioelectrical Impedance Analysis (BIA, BIA 101 Anniversary, Akern srl, Florence, Italy) according to the formula established by Janssen et al. (41) were assessed.

\section{Statistical analysis}

Descriptive statistical analysis comprised absolute frequencies and corresponding proportions, arithmetic means and median analyses. Between-group differences were assessed using Mann-Whitney-U-test. For longitudinal analyses Friedman's test was used with post-hoc Bonferroni correction. P values of less than 0.05 were considered statistically significant. All statistical analyses were performed using SPSS ver. 25 statistical software package (SPSS Inc. Chicago IL).

The study protocol was approved by the competent ethics committee at Wuerzburg University ( $\mathrm{N}$. 111/14) and registered with the German Register for Clinical Studies (DRKS00013262).

\section{Results}

Altogether 57 men were enrolled. There were 10 drop-outs before starting the intervention (3 due to acute illness, 7 didn't attend the sessions), so that eventually $n=47$ men accomplished the study. Mean age of all participants was 77.02 years (SD 6.1) at baseline. Of all $n=47$ patients, $n=11$ were randomized into the conventional RT group, $n=13$ in the WBV group, $\mathrm{n}=10$ in the QG group and $\mathrm{n}=13$ in the SO group. For a detailed overview on anthropometrics at baseline see also Table 1.

At baseline there were no significant differences between the groups, neither concerning anthropometric data, nor in terms of musculoskeletal function and strength. All $\mathrm{n}=47$ 
patients completed 6 months of training.

\section{Training adherence}

Overall mean training adherence was on average $\geq 75 \%$ for all groups together, with the highest adherence for the SO-group (85.2\%), then WBV group (83.2\%), then RT group (71.3\%) and the lowest for the QG group (65.1\%). All $\mathrm{n}=47$ patients completed 6 months of training and attended the 3 months interim analyses as well as the final assessment after 6 months.

Table 1: Means and SD for anthropometrics at baseline for all participants and exercise subgroups

\begin{tabular}{|c|c|c|c|c|c|}
\hline Mean (SD) & $\begin{array}{c}\text { All } \\
n=47\end{array}$ & $\begin{array}{c}\mathrm{RT} \\
\mathrm{n}=11\end{array}$ & $\begin{array}{l}\text { WBV } \\
n=13\end{array}$ & $\begin{array}{c}\text { QG } \\
n=10\end{array}$ & $\begin{array}{c}\mathrm{SO} \\
\mathrm{n}=13\end{array}$ \\
\hline Age (y) & $77.02(6.1)$ & $75.91(5.6)$ & $5.65(6.2)$ & $77.00(7.9)$ & $77.15(5.5)$ \\
\hline Height (cm) & $174.77(7.5)$ & $176.09(7.2)$ & 7.15 (6.7) & $174.40(9.3)$ & $177.23(6.7)$ \\
\hline Weight (kg) & $84.83(12.1)$ & $87.09(12.2)$ & $12.18(9.1)$ & $85.00(9.9)$ & $89.69(14.1)$ \\
\hline BMI (kg/mø) & $27.78(3.5)$ & $28.20(4.4)$ & $4.44(2.0)$ & $28.13(4.1)$ & 28.47 (3.4) \\
\hline SMI (kg/m】) & $10.19(1.0)$ & $10.60(1.3)$ & $1.33(0.7)$ & $10.18(0.9)$ & $9.96(0.9)$ \\
\hline
\end{tabular}

\section{Physical Performance}

Across all groups, average trunk strength increased substantially by $16.8 \%$ from $247.0 \mathrm{~nm}$ to $288.5 \mathrm{~nm}$ for TSE and by $10.7 \%$ from 136.0 to $150.5 \mathrm{~nm}$ for TSF. Comparing individual groups, this increase was most pronounced and statistically significant for TSE $(p=0.008)$ and TSF $(p=0.008)$ in the RT group. Trunk strength also increased in the SO and WBV group by TSE $+19.1 \%$ / TSF $+27.5 \%$ and TSE $+11.6 \%$ / TSF $+4.8 \%$, respectively, although these improvements were not statistically significant. In the QG group, trunk strength remained largely unaltered (also see Figure 2 and Figure 3).

Figure 2: Changes in trunk strength for extension in all 4 exercise groups

Figure 3: Changes in trunk strength for flexion in all 4 exercise groups 
HS improved significantly in the WBV group $(p=0.023)$ while there was no relevant change in all other groups. Regarding SB testing, none of the training interventions elicited a significant improvement. While USG increased significantly only in the RT $(p=0.015)$ group, improvements in the CRT were most pronounced and statistically significant in the SO and WBV groups. There were no significant changes in the overall SPPB-Score, the 6 MWT or the TUG test in any of the exercise groups scrutinized.

In addition, there was no significant change in the lumbar bending total range of motion (ROM) in any exercise group, even though improvements in this parameter and the 6MWT were borderline significant ( $\mathrm{p}=0.053$ and $\mathrm{p}=0.061$, respectively) in the QG group.

(For a detailed overview see table 2).

Constitutional parameters including height, weight and along with that BMI and SMI determined by BIA did not change significantly during the course of the study in any of the groups.

Comparative between group analyses considering the 4 different exercise regimens did not provide direct evidence that one exercise modality was in general superior to another with regards to generating muscle strength, function or muscle mass in comparison to the remaining ones.

\section{Safety and feasibility}

All 47 participants starting the exercise intervention adhered to the study protocol and concluded the study as intended. Moreover, throughout the conduct of the study, there were no adverse events associated with the interventions.

Table 2: Musculoskeletal assessment results in dependence of exercise modality (median/IQR - except mean/SD for SPPB and Balance)

Sig. assessment developments are in bold characters

\section{Subgroup analysis}

In order to evaluate training responses in participants at particularly high risk, subgroup analyses were performed considering the following three at-risk cohorts: All participants $\geq 80$ years of age $(\mathrm{n}=19 ; 40.4 \%$ of all participants), patients with low baseline SMI $\leq 10.75 \mathrm{~kg} / \mathrm{m} \square$ as assessed by BIA $(\mathrm{n}=30 ; 63.8 \%)$ and men with three or more chronic 
medical conditions ( $n=27 ; 57.4 \%$ ) fulfilling the definition of multimorbidity (42). Results for these subgroups were evaluated in an aggregated way irrespective of the assigned exercise group.

After 6 months of exercise intervention, the subgroup of patients $\geq 80$ years exhibited improvements in trunk strength which were statistically significant for TSF $(p=0.017)$. In addition, there was a significant reduction of the time required time to perform the TUG $(\mathrm{p}=0.036)$.

Participants with a reduced SMI of $\leq 10.75 \mathrm{~kg} / \mathrm{m} \square$ attained significant improvements for TSF $(p=0.035)$, HG $(p=0.026)$, CRT $(p=0.002)$ and UGS $(p=0.013)$ after 6 months of exercise intervention. Multimorbid men achieved significant improvements regarding TSE $(p=0.001)$ and TSF $(p=0.003)$, HG $(p=0.013)$ and CRT $(p=0.001)$ at the end of the study. Detailed results at baseline and at 3 and 6 months are provided in table 3, results for TSF are visualized in Fig 4.

Additional subgroup analyses within the at-risk groups did not reveal one exercise modality being superior above all others (data not shown).

Table 3: Musculoskeletal assessment results for subjects at high risk (median/IQR - except mean/SD for SPPB and Balance)

Sig. assessment developments are in bold characters

Figure 4: Changes in trunk strength for flexion in men at risk for muscular deficits

\section{Discussion}

To our knowledge this is the first prospective randomized study evaluating efficacy of alternative, less demanding training concepts in very old men at risk for osteoporosis and sarcopenia. Considering both transferability of the results into a real world setting, as well as applicability and feasibility in large, general population based studies, we selected three simple exercise modalities that neither require any previous sports experience nor a high level of motivation and compared these to professionally guided resistance exercise as a gold standard.

As expected, resistance exercise intervention clearly met the primary endpoint by yielding significant improvements in both trunk strength extension and flexion. This is in line with current literature (43) and reassuring in terms of the overall study setting and more importantly, it also confirms that appropriate 
exercise intervention can consistently improve muscular force and performance in a cohort of elderly men.

Even though improvements achieved with the three alternative interventions were not statistically significant regarding the primary endpoint, the extent of improvements and evaluation of secondary endpoints surely supports distinct beneficial effects for each of these concepts. Specifically, the improvement of trunk strength by $19.1 \%$ for extension and $27.5 \%$ for flexion by wearing a specific, flexible, customized off-the-shelf back brace is encouraging. Essentially, these results confirm previous reports stating that despite tenacious concerns about potential muscle atrophy due to trunk bracing, this particular device rather enhances muscle strength $(32-34,44)$. Similarly, WBV exercise over 6 months was also associated with non-significant but still considerable numerical improvements in trunk strength (TSE $+11.6 \%$ / TSF +4.8\%). In addition, both WBV and wearing the orthosis were associated with significant improvements regarding the time required to perform the CRT. While this has been reported before in conjunction with WBV exercise (45) and appears obvious considering how WBV exercise impacts thigh musculature, it is surely a novel and interesting finding with regards to the $S O$ that deserves further attention in forthcoming studies to see if this is due to and interventional effect extending further down the back including gluteal and/or ischiocrural musculature or if this is an indirect effect of encouraging subjects to be more active while wearing the brace. Not surprisingly, the QG group did not attain significant improvements in established measures of strength and power. Still, lumbar ROM improved numerically after 6 months and in line with previous literature, we also observed a trend to increased 6MWT distance (26).

In line with established principles for exercise intervention, it can be considered a general finding of this study, that improvements were observed in those functions specifically addressed by the respective intervention with no relevant off-target effects in the overall analysis, i.e. resistance exercise focusing on trunk strength is proficient in that regard without substantially improving other measures of physical performance. Accordingly WBV typically involving the thigh muscles and requiring the participant to firmly hold on to the handrail entails improvements in the CRT and HS while wearing a flexible, elastic back brace continuously stimulates and trains trunk muscles.

The overall small sample size is surely one of the main limitations of this pilot study that has to be kept in mind with regards to drawing further conclusions. Along with that, lack of statistical significance for several of the improvements and effects attained with regards to the primary and some key secondary endpoints is surely attributable to the piloting nature and small sample size of this study and this explanation in combination with some heterogeneity in baseline data also applies to the lack of significance for between group comparisons.

Importantly, all 4 training interventions were well accepted by the participants, notwithstanding them being on average 77 years old and generally not completely healthy. Specifically, adherence over time was particularly encouraging with all 47 subjects (100\%) completing the program. With regards to the available sessions attended, our result of $\geq 75 \%$ is in line with literature date reporting 58 to $77 \%$ (46). 
Similarly, the spinal orthosis was well accepted and perceived as being comfortable with $>80 \%$ of days meeting minimum wearing time. Main reasons for individual downtimes in wearing the back brace were acute health related issues and traveling.

Subgroup analyses revealed particularly pronounced and encouraging improvements for high risk subjects (Age $\geq 80$ years, $\mathrm{SMI} \leq 10.75 \mathrm{~kg} / \mathrm{m}^{2}, \geq 3$ chronic diseases) irrespective of the applied intervention, i.e. specifically patients at risk and with severe health restrictions benefit from any kind of intervention and all 3 vulnerable subgroups achieved significant improvements specifically in their lumbar flexion strength, various lower extremity performance measures (CRT, TUG, 6MW) and HS.

Taken together, these results indicate that the low impact training-concepts scrutinized in this study are particularly suitable for very old, multimorbid and sarcopenic subjects with restricted mobility and elevated fracture risk. Future studies with more participants are needed to further substantiate these results. Data presented here should be considered a starting point for targeted evaluations if and how these simple exercise modalities can be effectively implemented in routine care for long term prevention of age associated functional decline.

\section{Conclusions}

Specific exercise interventions lead to explicit functional improvements even in very old men. For very old, week and ill patients, even simple exercise interventions not requiring a high level of commitment can be beneficial.

\section{Declarations}

\section{Ethics approval and consent to participate:}

The study protocol was approved by the competent ethics committee at Wuerzburg University (N. 111/14).

Every participant gave written informed consent to participate and for publication before any study related procedures.

Availability of data and materials: All data and study material is stored at the Departments of orthopedics at the University of Wuerzburg for 10 years. The datasets used and analyzed during the current study are available from the corresponding author on reasonable request

\section{Competing Interests / Disclosures}

LS has received honoraria for lectures and advice from Abbvie, Amgen, Alexion, KyowaKirin, Lilly, Medi, MSD, Novartis, Servier and UCB and research grants to the Institution (University of Wuerzburg) from Alexion, Kyowa Kirin and Novartis. 
FG has received honoraria for lectures from Abbvie, Alexion and Lilly.

SL, SS, and MS report no COI during the course of the study.

Funding: Parts of this work have been supported by the Bayerische Forschungsstiftung, Munich Germany, Predia Institute, Wuerzburg, Germany; medi Bayreuth, Bayreuth, Germany, SMT medical, Wuerzburg, Germany; Novotec Medical, Pforzheim, Germany

\section{Author contributions:}

Study design: LS

Study lead investigator: LS

Enrolled and studied patients: LS, FG

Collection and assembly of data: FG, SL, SS, MS

Data analysis: LS, FG,

Data interpretation: LS, FG

Manuscript preparation: LS, FG

Manuscript content review and revisions: All authors.

Approval of the final Manuscript: All authors

\section{Acknowledgements}

The authors acknowledge Prof. Franz Jakob for organizational support and the Bayerische Forschungsstiftung, Munich, Germany; Predia Institute, Wuerzburg, Germany; medi Bayreuth, Bayreuth, Germany, SMT medical, Wuerzburg, Germany; Novotec Medical, Pforzheim, Germany for supporting and funding aspects of this study.

\section{Abbreviations}

TSE - $\quad$ Trunk strength for extension

TSF - $\quad$ Trunk strength for flexion

HS $\quad$ - $\quad$ Handgrip strength

CRT - $\quad$ Chair Rise Test

UGS - $\quad$ Usual Gait Speed 


$\begin{array}{lll}\text { SB } & - & \text { Static Balance } \\ \text { TUG } & - & \text { Timed Up and Go test } \\ \text { SPPB } & - & \text { Short Physical Performance Battery } \\ \text { 6MW } & - & 6 \text { Minute Walk test } \\ \text { RT } & - & \text { Resistance Training Group } \\ \text { QG } & - & \text { Qi Gong Training Group } \\ \text { SO } & - & \text { Spinal Orthosis Training Group } \\ \text { WBV } & - & \text { Whole Body Vibration Training Group } \\ \text { SMI } & - & \text { Skeletal Muscle Index } \\ \text { ROM } & - & \text { Range of Motion } \\ \text { BIA } & - & \text { Bioelectrical Impedance analysis }\end{array}$

\section{References}

1. Cruz-Jentoft AJ, Baeyens JP, Bauer JM, Boirie Y, Cederholm T, Landi F, et al. Sarcopenia: European consensus on definition and diagnosis: Report of the European Working Group on Sarcopenia in Older People. Age Ageing. 2010;39(4):412-23.

2. Cruz-Jentoft AJ, Landi F, Topinkova E, Michel JP. Understanding sarcopenia as a geriatric syndrome. Curr Opin Clin Nutr Metab Care. 2010;13(1):1-7.

3. Hirschfeld HP, Kinsella R, Duque G. Osteosarcopenia: where bone, muscle, and fat collide. Osteoporos Int. 2017;28(10):2781-90.

4. Fischer S, Kapinos KA, Mulcahy A, Pinto L, Hayden O, Barron R. Estimating the long-term functional burden of osteoporosis-related fractures. Osteoporos Int. 2017;28(10):2843-51.

5. Landi F, Liperoti R, Russo A, Giovannini S, Tosato M, Capoluongo E, et al. Sarcopenia as a risk factor for falls in elderly individuals: results from the ilSIRENTE study. Clinical nutrition (Edinburgh, Scotland). 2012;31(5):652-8.

6. Uemura K, Makizako H, Lee S, Doi T, Lee S, Tsutsumimoto K, et al. The impact of sarcopenia on incident homebound status among community-dwelling older adults: A prospective cohort study. Maturitas. 2018;113:26-31.

7. Van Der Klift M, Pols HA, Geleijnse JM, Van Der Kuip DA, Hofman A, De Laet CE. Bone mineral density and mortality in elderly men and women: the Rotterdam Study. Bone. 2002;30(4):643-8. 
8. Scott D, Seibel M, Cumming R, Naganathan V, Blyth F, Le Couteur DG, et al. Sarcopenic Obesity and Its Temporal Associations With Changes in Bone Mineral Density, Incident Falls, and Fractures in Older Men: The Concord Health and Ageing in Men Project. J Bone Miner Res. 2017;32(3):575-83.

9. Cruz-Jentoft AJ, Bahat G, Bauer J, Boirie Y, Bruyere O, Cederholm T, et al. Sarcopenia: revised European consensus on definition and diagnosis. Age and ageing. 2019;48(1):16-31.

10. Landi F, Calvani R, Cesari M, Tosato M, Martone AM, Ortolani E, et al. Sarcopenia: An Overview on Current Definitions, Diagnosis and Treatment. Curr Protein Pept Sci. 2018;19(7):633-8.

11. Chalhoub D, Cawthon PM, Ensrud KE, Stefanick ML, Kado DM, Boudreau R, et al. Risk of Nonspine Fractures in Older Adults with Sarcopenia, Low Bone Mass, or Both. J Am Geriatr Soc. 2015;63(9):1733-40.

12. Zanker J, Duque G. Osteosarcopenia: the Path Beyond Controversy. Curr Osteoporos Rep. 2020.

13. Wagner P, Chapurlat R, Ecochard R, Szulc P. Low Muscle Strength and Mass Is Associated With the Accelerated Decline of Bone Microarchitecture at the Distal Radius in Older Men: the Prospective STRAMBO Study. J Bone Miner Res. 2018;33(9):1630-40.

14. Tarantino U, Baldi J, Scimeca M, Piccirilli E, Piccioli A, Bonanno E, et al. The role of sarcopenia with and without fracture. Injury. 2016;47 Suppl 4:S3-S10.

15. Buehring B, Hansen KE, Lewis BL, Cummings SR, Lane NE, Binkley N, et al. Dysmobility Syndrome Independently Increases Fracture Risk in the Osteoporotic Fractures in Men (MrOS) Prospective Cohort Study. J Bone Miner Res. 2018;33(9):1622-9.

16. Ebeling PR, Cicuttini F, Scott D, Jones G. Promoting mobility and healthy aging in men: a narrative review. Osteoporos Int. 2019;30(10):1911-22.

17. Nawrat-Szoltysik AJ, Polak A, Malecki A, Piejko L, Grzybowska-Ganszczyk D, Krecichwost M, et al. Effect of physical activity on the sequelae of osteoporosis in female residents of residential care facilities. Advances in clinical and experimental medicine : official organ Wroclaw Medical University. 2018;27(5):633-42.

18. Wisdom KM, Delp SL, Kuhl E. Use it or lose it: multiscale skeletal muscle adaptation to mechanical stimuli. Biomech Model Mechanobiol. 2015;14(2):195-215.

19. Cesari M, Vellas B, Hsu FC, Newman AB, Doss $H$, King AC, et al. A physical activity intervention to treat the frailty syndrome in older persons-results from the LIFE-P study. J Gerontol A Biol Sci Med Sci. 2015;70(2):216-22.

20. Cadore EL, Casas-Herrero A, Zambom-Ferraresi F, Idoate F, Millor N, Gomez M, et al. Multicomponent exercises including muscle power training enhance muscle mass, power output, and functional outcomes in institutionalized frail nonagenarians. Age. 2014;36(2):773-85.

21. Chen HT, Wu HJ, Chen YJ, Ho SY, Chung YC. Effects of 8-week kettlebell training on body composition, muscle strength, pulmonary function, and chronic low-grade inflammation in elderly women with sarcopenia. Exp Gerontol. 2018;112:112-8.

22. Moore DR, Kelly RP, Devries MC, Churchward-Venne TA, Phillips SM, Parise G, et al. Low-load resistance exercise during inactivity is associated with greater fibre area and satellite cell expression 
in older skeletal muscle. J Cachexia Sarcopenia Muscle. 2018;9(4):747-54.

23. Seldeen KL, Lasky G, Leiker MM, Pang M, Personius KE, Troen BR. High Intensity Interval Training Improves Physical Performance and Frailty in Aged Mice. J Gerontol A Biol Sci Med Sci. 2018;73(4):429-37.

24. Moore SA, Hrisos N, Errington L, Rochester L, Rodgers $H$, Witham $M$, et al. Exercise as a treatment for sarcopenia: an umbrella review of systematic review evidence. Physiotherapy. 2019;107:189-201.

25. Sherrington C, Fairhall NJ, Wallbank GK, Tiedemann A, Michaleff ZA, Howard K, et al. Exercise for preventing falls in older people living in the community. The Cochrane database of systematic reviews. 2019;1:Cd012424.

26. Pippa L, Manzoli L, Corti I, Congedo G, Romanazzi L, Parruti G. Functional capacity after traditional Chinese medicine (qi gong) training in patients with chronic atrial fibrillation: a randomized controlled trial. Preventive cardiology. 2007;10(1):22-5.

27. Song Q, Li L, Zhang C, Sun W, Mao D. Long-term Tai Chi practitioners have superior body stability under dual task condition during stair ascent. Gait Posture. 2018;66:124-9.

28. Vergara-Diaz G, Osypiuk K, Hausdorff JM, Bonato P, Gow BJ, Miranda JG, et al. Tai Chi for Reducing Dual-task Gait Variability, a Potential Mediator of Fall Risk in Parkinson's Disease: A Pilot Randomized Controlled Trial. Global advances in health and medicine. 2018;7:2164956118775385.

29. Seefried L, Genest F, Luksche N, Schneider M, Fazeli G, Brandl M, et al. Efficacy and safety of whole body vibration in maintenance hemodialysis patients - A pilot study. J Musculoskelet Neuronal Interact. 2017;17(4):268-74.

30. Bogaerts A, Verschueren S, Delecluse C, Claessens AL, Boonen S. Effects of whole body vibration training on postural control in older individuals: a 1 year randomized controlled trial. Gait Posture. 2007;26(2):309-16.

31. Bautmans I, Van Hees E, Lemper JC, Mets T. The feasibility of Whole Body Vibration in institutionalised elderly persons and its influence on muscle performance, balance and mobility: a randomised controlled trial [ISRCTN62535013]. BMC Geriatr. 2005;5:17.

32. Dionyssiotis Y, Trovas G, Thoma S, Lyritis G, Papaioannou N. Prospective study of spinal orthoses in women. Prosthetics and orthotics international. 2015;39(6):487-95.

33. Valentin GH, Pedersen LN, Maribo T. Wearing an active spinal orthosis improves back extensor strength in women with osteoporotic vertebral fractures. Prosthetics and orthotics international. 2014;38(3):232-8.

34. Pfeifer M, Kohlwey L, Begerow B, Minne HW. Effects of two newly developed spinal orthoses on trunk muscle strength, posture, and quality-of-life in women with postmenopausal osteoporosis: a randomized trial. Am J Phys Med Rehabil. 2011;90(10):805-15.

35. Kaijser Alin C, Uzunel E, Grahn Kronhed AC, Alinaghizadeh H, Salminen H. Effect of treatment on back pain and back extensor strength with a spinal orthosis in older women with osteoporosis: a randomized controlled trial. Archives of osteoporosis. 2019;14(1):5. 
36. Pfeil A, Lehmann G, Lange U. [Update DVO guidelines 2017 on "Prophylaxis, diagnostics and treatment of osteoporosis in postmenopausal women and men": What is new, what remains for rheumatologists?]. Z Rheumatol. 2018.

37. Neuerburg C, Mittlmeier L, Schmidmaier R, Kammerlander C, Bocker W, Mutschler W, et al. Investigation and management of osteoporosis in aged trauma patients: a treatment algorithm adapted to the German guidelines for osteoporosis. Journal of orthopaedic surgery and research. 2017;12(1):86.

38. Guralnik JM, Simonsick EM, Ferrucci L, Glynn RJ, Berkman LF, Blazer DG, et al. A short physical performance battery assessing lower extremity function: association with self-reported disability and prediction of mortality and nursing home admission. Journal of gerontology. 1994;49(2):M85-94.

39. Cruz-Jentoft AJ, Baeyens JP, Bauer JM, Boirie Y, Cederholm T, Landi F, et al. Sarcopenia: European consensus on definition and diagnosis: Report of the European Working Group on Sarcopenia in Older People. Age and ageing. 2010;39(4):412-23.

40. Mathias S, Nayak US, Isaacs B. Balance in elderly patients: the "get-up and go" test. Arch Phys Med Rehabil. 1986;67(6):387-9.

41. Janssen I, Heymsfield SB, Baumgartner RN, Ross R. Estimation of skeletal muscle mass by bioelectrical impedance analysis. J Appl Physiol (1985). 2000;89(2):465-71.

42. Schafer I, Hansen H, Schon G, Maier W, Hofels S, Altiner A, et al. The German MultiCare-study: Patterns of multimorbidity in primary health care - protocol of a prospective cohort study. BMC Health Serv Res. 2009;9:145.

43. Lichtenberg T, von Stengel S, Sieber C, Kemmler W. The Favorable Effects of a High-Intensity Resistance Training on Sarcopenia in Older Community-Dwelling Men with Osteosarcopenia: The Randomized Controlled FrOST Study. Clin Interv Aging. 2019;14:2173-86.

44. Li M, Law SW, Cheng J, Kee HM, Wong MS. A comparison study on the efficacy of SpinoMed(R) and soft lumbar orthosis for osteoporotic vertebral fracture. Prosthetics and orthotics international. 2015;39(4):270-6.

45. Beck BR, Norling TL. The effect of 8 mos of twice-weekly low- or higher intensity whole body vibration on risk factors for postmenopausal hip fracture. Am J Phys Med Rehabil. 2010;89(12):9971009.

46. Picorelli AM, Pereira LS, Pereira DS, Felício D, Sherrington C. Adherence to exercise programs for older people is influenced by program characteristics and personal factors: a systematic review. Journal of physiotherapy. 2014;60(3):151-6.

\section{Tables 2 And 3}

Table 2: Musculoskeletal assessment results in dependence of exercise modality (median/IQR - except mean/SD for SPPB and Balance) 


\begin{tabular}{|c|c|c|c|c|}
\hline Median (IQR) & 0 months & 3 months & 6 months & p-value \\
\hline Lumbar Extension (nm). & - & - & - & - \\
\hline Overall & $247.0(117.0)$ & $267.0(93.0)$ & $288.5(113.0)$ & 0.003 \\
\hline Qi Gong & 252.0 (92.5) & $254.0(110.3)$ & $248.0(155.0)$ & 0.417 \\
\hline Resistance Training & $240.0(171.8)$ & $320.0(156.0)$ & $333.5(154.3)$ & 0.008 \\
\hline Whole Body Vibration & $241.0(101.0)$ & $245.0(49.0)$ & $269.0(55.8)$ & 0.103 \\
\hline Lumbar Orthosis & $269.0(92.5)$ & $251.0(110.25)$ & $320.5(155.0)$ & 0.223 \\
\hline Lumbar Flexion (nm) & - & - & - & - \\
\hline Overall & $136.0(48.5)$ & $137.0(54.0)$ & $150.5(62.3)$ & $<0.001$ \\
\hline Qi Gong & $143.0(90.0)$ & $162.5(103.5)$ & $143.0(130.5)$ & 0.236 \\
\hline Resistance Training & $135.0(51.5)$ & $137.0(69.0)$ & $178.0(76.0)$ & 0.008 \\
\hline Whole Body Vibration & $136.0(36.0)$ & $132.0(38.0)$ & $142.5(30.5)$ & 0.115 \\
\hline Spinal Orthosis & $129.0(90.0)$ & $144.0(103.5)$ & $164.5(130.5)$ & 0.072 \\
\hline Lumbar Ext/Flex. total ROM (- ${ }_{-}^{\circ}$. & - & - & - & - \\
\hline Overall & $50.0(14.5)$ & $55.0(18.5)$ & $53.5(16.5)$ & 0.951 \\
\hline Qi Gong & $47.0(19.0)$ & $45.0(25.8)$ & $49.5(16.0)$ & 0.053 \\
\hline Resistance Training & $57.50(22.3)$ & $60.0(19.0)$ & $60.5(14.3)$ & 0.135 \\
\hline Whole Body Vibration & $56.0(29.5)$ & $55.0(20.0)$ & $54.5(15.0)$ & 0.931 \\
\hline Spinal Orthosis & $48.0(19.0)$ & $56.0(25.8)$ & $48.5(16.0)$ & 0.072 \\
\hline Grip Strength (kg). & - & - & - & - \\
\hline Overall & $32.4(9.2)$ & $33.1(10.2)$ & $32.9(8.7)$ & 0.006 \\
\hline Qi Gong & $27.0(17.6)$ & $27.65(14.4)$ & $29.0(14.3)$ & 0.122 \\
\hline Resistance Training & $34.6(7.5)$ & $37.4(11.1)$ & $32.9(5.7)$ & 0.178 \\
\hline Whole Body Vibration & $31.9(6.7)$ & $32.5(8.8)$ & $32.8(6.3)$ & 0.023 \\
\hline Spinal Orthosis & $33.8(17.6)$ & $35.5(14.4)$ & $34.2(14.4)$ & 0.794 \\
\hline Balance overall (sec). & - & - & - & - \\
\hline Overall & $27.9(4.7)$ & $27.8(4.8)$ & $27.3(6.0)$ & 0.738 \\
\hline Qi Gong & $27.1(6.2)$ & $27.3(4.5)$ & $26.2(6.6)$ & 0.601 \\
\hline Resistance Training & $27.0(6.2)$ & $27.2(4.0)$ & $27.1(4.3)$ & 0.991 \\
\hline Whole Body Vibration & $27.8(4.1)$ & $28.8(2.2)$ & $29.7(0.9)$ & 0.177 \\
\hline Spinal Orthosis & $29.3(2.0)$ & $27.6(7.2)$ & $26.1(8.9)$ & 0.283 \\
\hline Gait Speed (m/s). & - & - & - & - \\
\hline Overall & $1.22(0.2)$ & $1.18(0.3)$ & $1.25(0.2)$ & 0.023 \\
\hline
\end{tabular}




\begin{tabular}{|c|c|c|c|c|}
\hline Qi Gong & $1.25(0.4)$ & $1.18(0.3)$ & $1.20(0.2)$ & 0.452 \\
\hline Resistance Training & $1.22(0.1)$ & $1.32(0.3)$ & $1.32(0.3)$ & 0.015 \\
\hline Whole Body Vibration & $1.22(0.2)$ & $1.18(0.3)$ & $1.26(0.2)$ & 0.942 \\
\hline Spinal Orthosis & $1.16(0.4)$ & $1.07(0.3)$ & $1.23(0.2)$ & 0.199 \\
\hline$\underline{C R T}(\underline{s e c})$. & - & - & - & - \\
\hline Overall & $8.81(2.6)$ & $8.96(2.3)$ & $8.03(2.6)$ & $<0.001$ \\
\hline Qi Gong & $9.27(3.8)$ & $9.86(6.5)$ & $8.70(5.2)$ & 0.497 \\
\hline Resistance Training & $8.18(2.9)$ & $8.69(2.1)$ & $8.14(2.0)$ & 0.441 \\
\hline Whole Body Vibration & $8.81(2.3)$ & $8.57(1.8)$ & $8.03(2.0)$ & 0.007 \\
\hline Spinal Orthosis & $8.62(3.8)$ & $10.28(6.5)$ & $7.84(5.2)$ & 0.003 \\
\hline SPPB Score (points). & - & - & - & - \\
\hline Overall & $11.2(1.6)$ & $11.1(4.6)$ & $11.3(1.3)$ & 0.743 \\
\hline Qi Gong & $10.9(2.0)$ & $10.8(1.8)$ & $10.9(1.9)$ & 0.927 \\
\hline Resistance Training & $11.3(1.6)$ & $11.4(1.0)$ & $11.3(1.1)$ & 0.959 \\
\hline Whole Body Vibration & $11.3(1.1)$ & $11.7(0.5)$ & $11.8(0.4)$ & 0.163 \\
\hline Spinal Orthosis & $11.1(1.8)$ & $10.5(2.3)$ & $11.3(1.6)$ & 0.424 \\
\hline$\underline{6 M W}(\underline{\underline{m}})$ & - & - & - & - \\
\hline Overall & $544.0(127.0)$ & $548.0(122.0)$ & $524.0(154.0)$ & 0.017 \\
\hline Qi Gong & $553.0(189.8)$ & $579.5(179.0)$ & $572.0(193.0)$ & 0.061 \\
\hline Resistance Training & $590.0(240.0)$ & $548.0(127.0)$ & $524.0(154.0)$ & 0.076 \\
\hline Whole Body Vibration & $544.0(118.5)$ & $549.0(118.0)$ & $512.0(180.0)$ & 0.735 \\
\hline Spinal Orthosis & $528.0(189.8)$ & $538.0(179.0)$ & $527.0(193.0)$ & 0.484 \\
\hline$\underline{T U G}(\underline{s e c})$. & - & - & - & - \\
\hline Overall & $8.31(2.6)$ & $8.09(1.8)$ & $8.16(8.2)$ & 0.273 \\
\hline Qi Gong & $8.10(3.2)$ & $8.28(4.3)$ & $8.19(3.4)$ & 0.741 \\
\hline Resistance Training & $8.31(1.9)$ & $7.87(2.4)$ & $7.91(1.2)$ & 0.307 \\
\hline Whole Body Vibration & $8.18(2.9)$ & $7.71(0.9)$ & $7.97(1.3)$ & 0.232 \\
\hline Spinal Orthosis & $8.56(3.2)$ & $8.81(4.3)$ & $9.00(6.2)$ & 0.368 \\
\hline
\end{tabular}

Sig. assessment developments are in bold characters

Table 3: Musculoskeletal assessment results for subjects at high risk (median/IQR - except mean/SD for SPPB and Balance) 


\begin{tabular}{|c|c|c|c|c|}
\hline Median (IQR) & 0 months & 3 months & 6 months & p-value \\
\hline Lumbar Extension (nm). & - & - & - & - \\
\hline Men $\geq 80$ years & $241.0(111.0)$ & $238.0(89.5)$ & $249.0(92.0)$ & 0.185 \\
\hline $\mathrm{SMI} \leq 10.75 \mathrm{~kg} / \mathrm{m} \square$ & $247.0(90.0)$ & $259.0(61.5)$ & $290.0(102.0)$ & 0.093 \\
\hline Multimorbid men & $246.5(149.8)$ & $256.5(106.8)$ & $300.5(131.3)$ & $<0.001$ \\
\hline Lumbar Flexion (nm) & - & - & - & - \\
\hline Men $\geq 80$ years & $125.0(51.0)$ & $105.5(45.3)$ & $139.0(31.0)$ & 0.017 \\
\hline $\mathrm{SMI} \leq 10.75 \mathrm{~kg} / \mathrm{m} \square$ & $129.0(53.0)$ & $133.0(51.5)$ & $141.0(74.0)$ & 0.035 \\
\hline Multimorbid men & $127.0(65.0)$ & $133.0(60.5)$ & $153.0(75.8)$ & 0.001 \\
\hline Lumbar ROM ( $\left.{ }_{-}^{\circ}\right)$ & - & - & - & - \\
\hline Men $\geq 80$ years & $48.0(20.0)$ & $54.5(13.5)$ & $53.0(15.5)$ & 0.611 \\
\hline $\mathrm{SMI} \leq 10.75 \mathrm{~kg} / \mathrm{m} \square$ & $54.0(17.5)$ & $55.5(20.0)$ & $54.0(20.0)$ & 0.738 \\
\hline Multimorbid men & $47.0(17.3)$ & $52.0(14.3)$ & $52.0(15.5)$ & 0.963 \\
\hline Grip Strength (kg). & - & - & - & - \\
\hline Men $\geq 80$ years & $29.4(6.5)$ & $29.2(5.9)$ & $30.2(7.9)$ & 0.076 \\
\hline $\mathrm{SMI} \leq 10.75 \mathrm{~kg} / \mathrm{m} \square$ & $31.6(9.2)$ & $33.3(10.1)$ & $32.8(7.9)$ & 0.026 \\
\hline Multimorbid men & $29.4(9.3)$ & $30.2(10.2)$ & $32.9(9.0)$ & 0.034 \\
\hline Balance overall (sec) & - & - & - & - \\
\hline Men $\geq 80$ years & $25.9(6.3)$ & $26.1(6.5)$ & $23.9(8.3)$ & 0.353 \\
\hline $\mathrm{SMI} \leq 10.75 \mathrm{~kg} / \mathrm{m} \square$ & $28.2(1.7)$ & $27.7(1.8)$ & $26.8(1.5)$ & 0.368 \\
\hline Multimorbid men & $27.5(5.6)$ & $27.5(5.7)$ & $26.0(7.5)$ & 0.338 \\
\hline Gait Speed $(\underline{m / s})$ & - & - & - & - \\
\hline Men $\geq 80$ years & $1.22(0.3)$ & $1.13(0.2)$ & $1.16(0.2)$ & 0.750 \\
\hline $\mathrm{SMI} \leq 10.75 \mathrm{~kg} / \mathrm{m} \square$ & $1.22(0.2)$ & $1.17(0.3)$ & $1.23(0.2)$ & 0.109 \\
\hline Multimorbid men & $1.22(0.2)$ & $1.19(0.3)$ & $1.23(0.2)$ & 0.074 \\
\hline$\underline{C R T}(\underline{s e c})$ & - & - & - & - \\
\hline Men $\geq 80$ years & $10.27(2.3)$ & $9.50(3.1)$ & $8.95(3.1)$ & 0.092 \\
\hline $\mathrm{SMI} \leq 10.75 \mathrm{~kg} / \mathrm{m} \square$ & $8.66(2.4)$ & $8.97(2.5)$ & $7.99(3.0)$ & 0.002 \\
\hline Multimorbid men & $8.81(3.5)$ & $8.66(2.2)$ & $8.14(3.6)$ & 0.004 \\
\hline SPPB Score (points) & - & - & - & - \\
\hline Men $\geq 80$ years & $10.6(1.8)$ & $10.6(1.9)$ & $10.7(1.5)$ & 0.627 \\
\hline $\mathrm{SMI} \leq 10.75 \mathrm{~kg} / \mathrm{m} \square$ & $11.1(4.1)$ & $11.0(5.4)$ & $11.2(7.0)$ & 0.972 \\
\hline Multimorbid men & $10.8(1.9)$ & $11.0(1.9)$ & $11.0(1.6)$ & 0.233 \\
\hline
\end{tabular}




\begin{tabular}{|c|c|c|c|c|}
\hline$\underline{6 M W}(\underline{m})$. & - & - & - & - \\
\hline Men $\geq 80$ years & $528.0(100.0)$ & $529.0(129.0)$ & $512.0(179.0)$ & 0.331 \\
\hline $\mathrm{SMI} \leq 10.75 \mathrm{~kg} / \mathrm{m}$ & $544.0(120.5)$ & $552.0(128.5)$ & $541.0(160.5)$ & 0.059 \\
\hline Multimorbid men & $544.0(133.0)$ & $538.0(93.0)$ & $510.0(145.0)$ & 0.034 \\
\hline$\underline{T U G}(\underline{s e c})$ & - & - & - & - \\
\hline Men $\geq 80$ years & $9.78(3.1)$ & $8.38(2.1)$ & $8.69(3.1)$ & 0.036 \\
\hline $\mathrm{SMI} \leq 10.75 \mathrm{~kg} / \mathrm{m}$ & $8.41(3.3)$ & $8.31(1.5)$ & $8.33(2.1)$ & 0.273 \\
\hline Multimorbid men & $8.22(3.3)$ & $7.87(1.8)$ & $8.16(1.9)$ & 0.163 \\
\hline
\end{tabular}

Sig. assessment developments are in bold characters

\section{Figures}

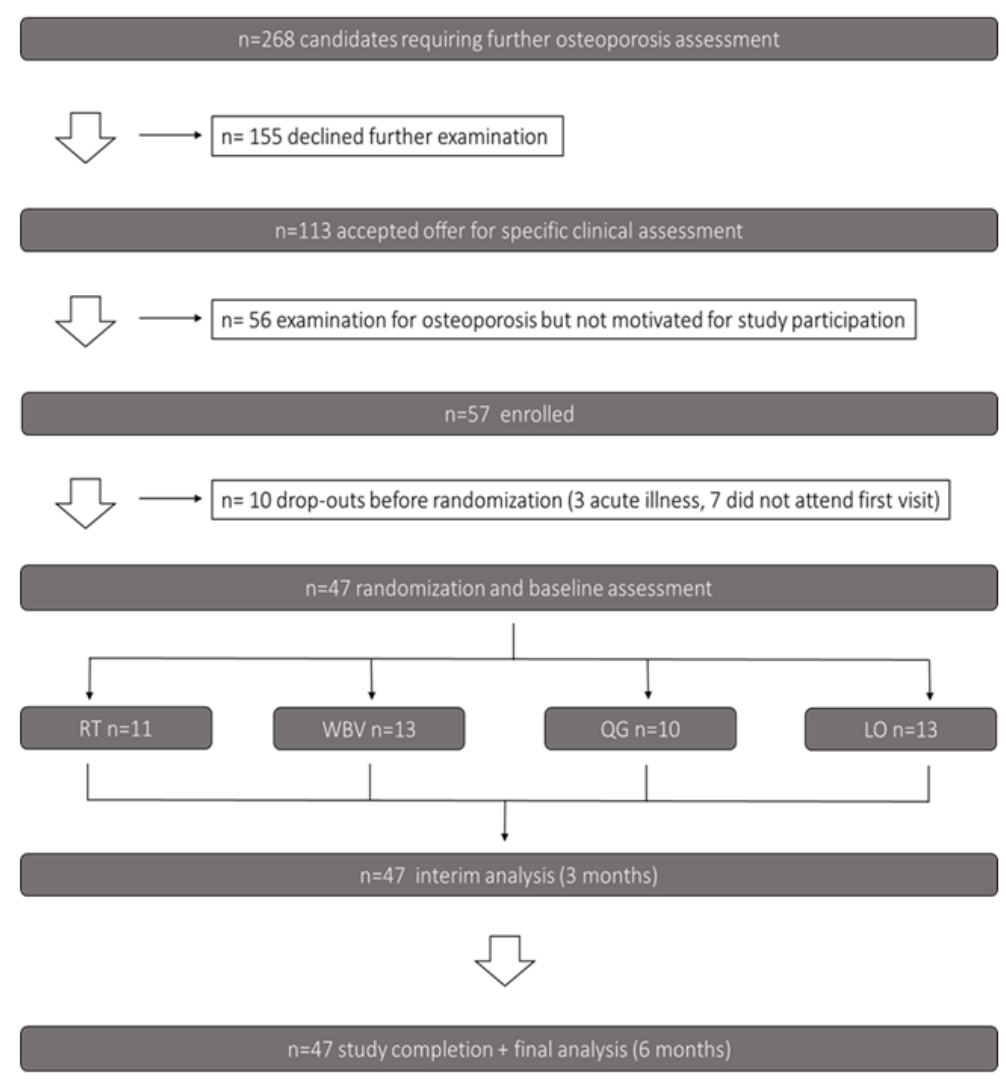

Figure 1

Study population flow 


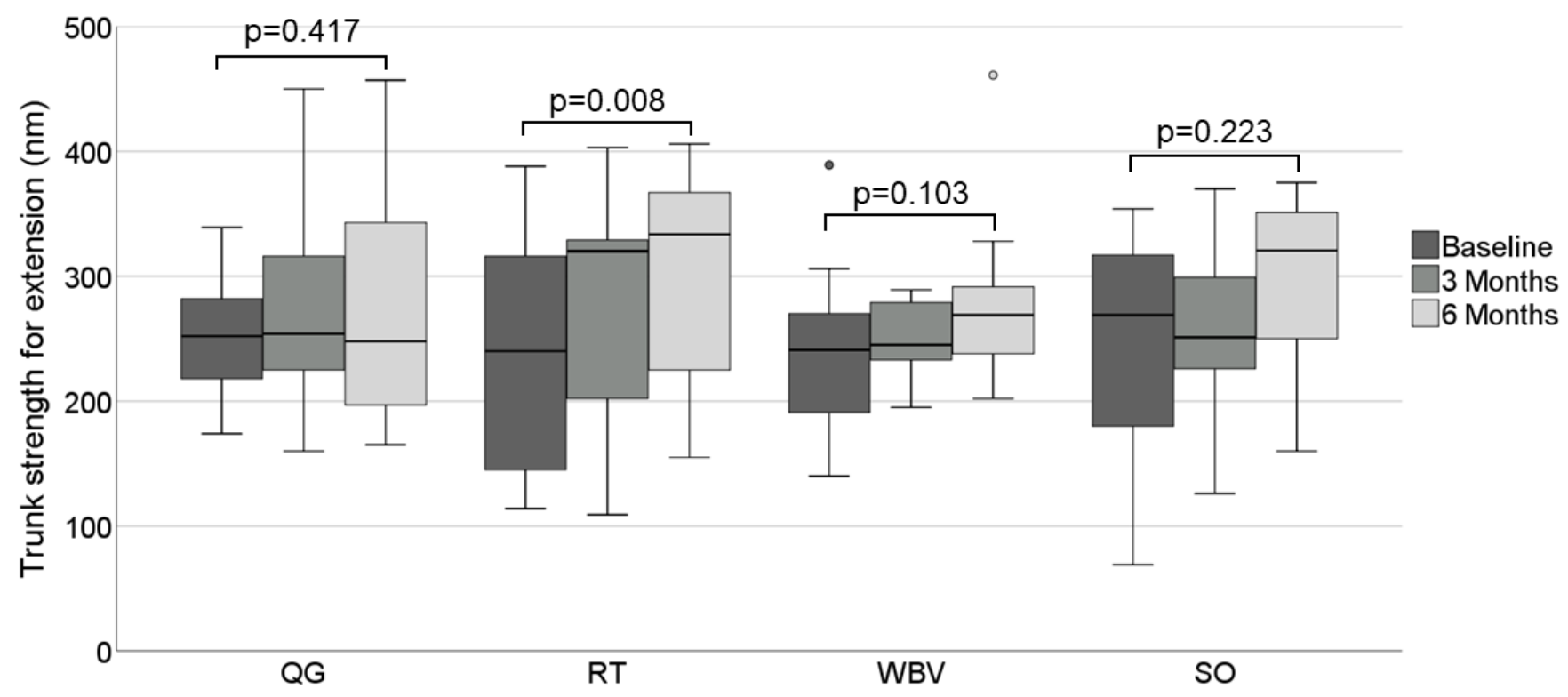

Exercise Group

Figure 2

Changes in trunk strength for extension in all 4 exercise groups

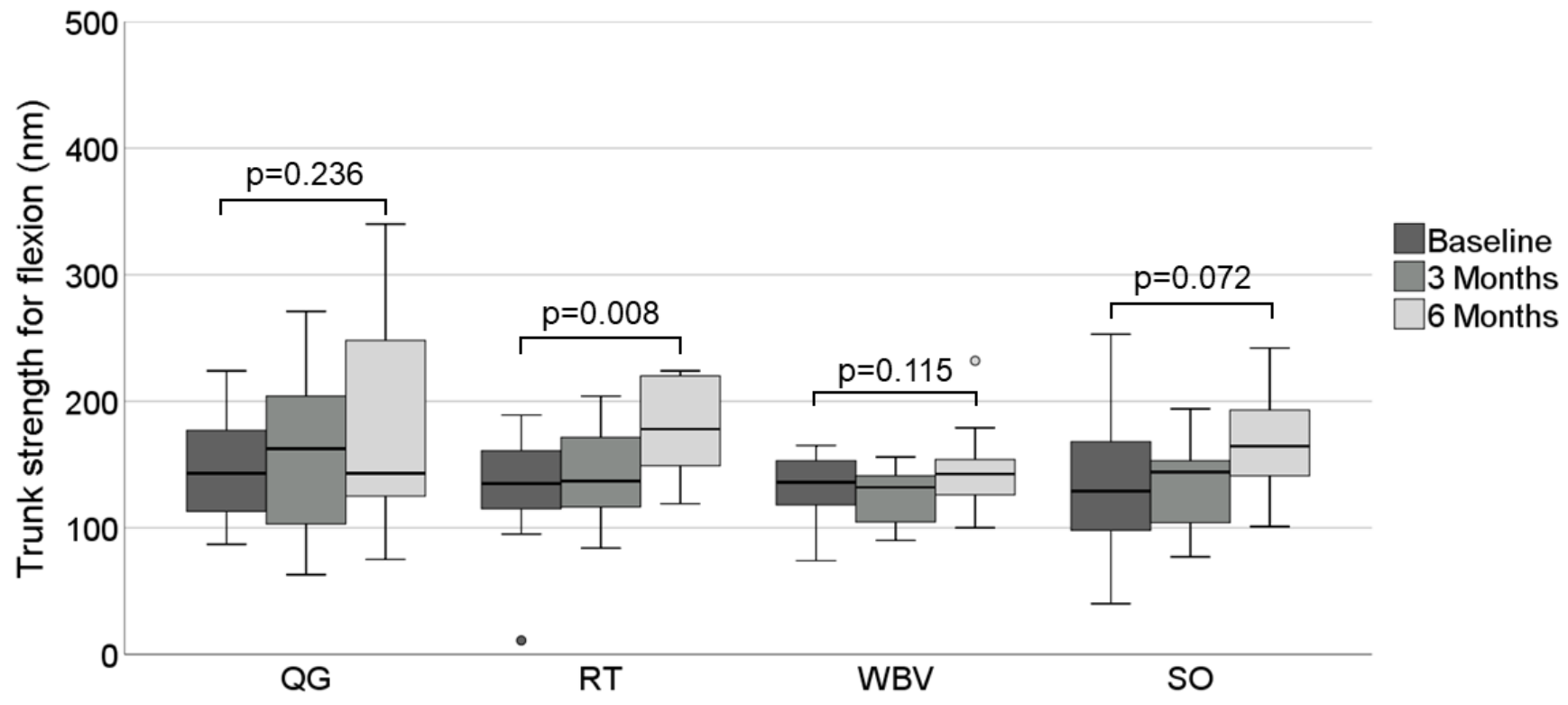

Exercise Group 
Figure 3

Changes in trunk strength for flexion in all 4 exercise groups

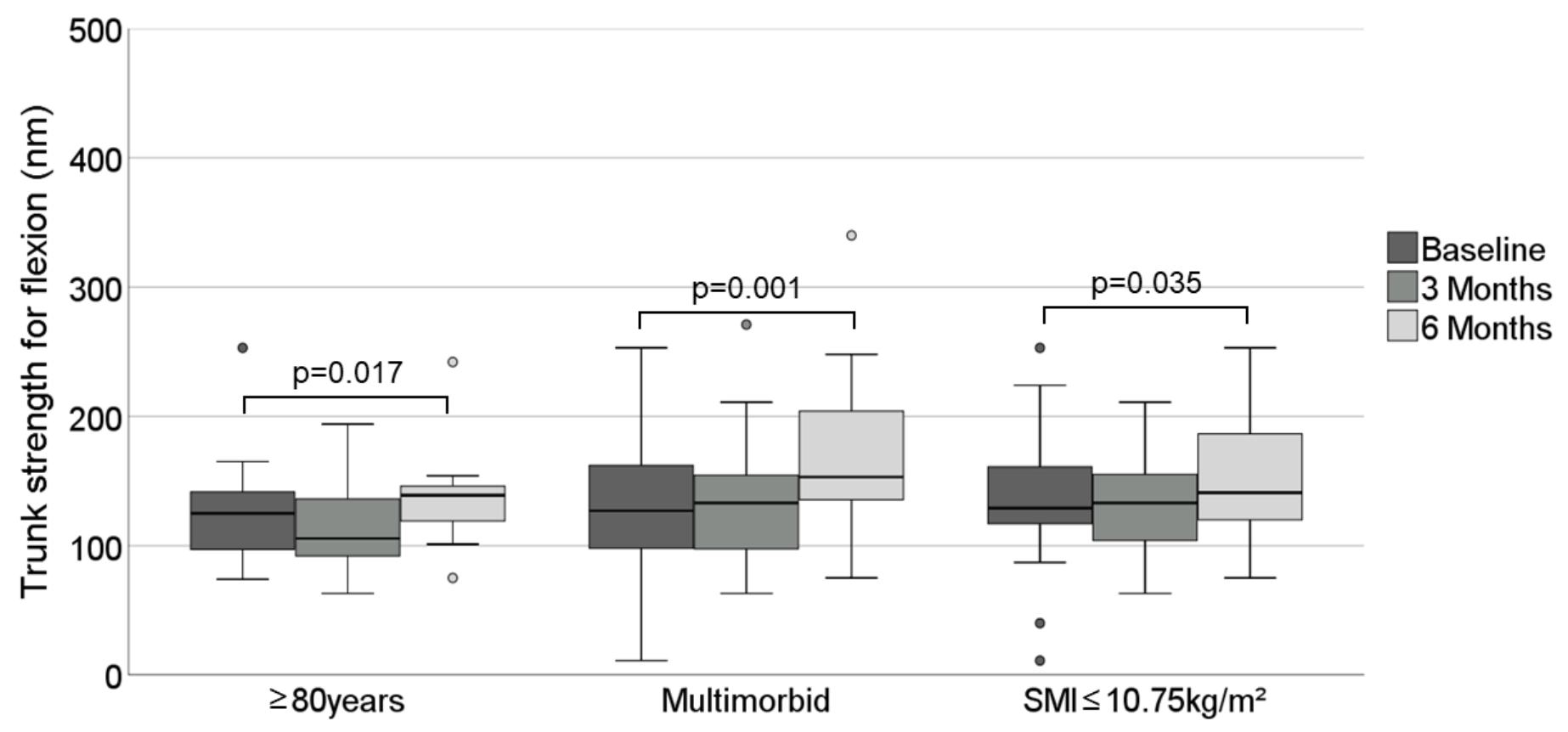

Vulnerable patients

\section{Figure 4}

Changes in trunk strength for flexion in men at risk for muscular deficits 